\title{
Spectroscopic study of blue compact galaxies
}

\section{The spectra`}

\author{
X. Kong ${ }^{1,2,3}$ and F. Z. Cheng ${ }^{2,3}$ \\ 1 Max Planck Institute for Astrophysics, Karl-Schwarzschild-Str. 1, 85741 Garching, Germany \\ 2 Center for Astrophysics, University of Science and Technology of China, 230026, Hefei, PR China \\ 3 National Astronomical Observatories, Chinese Academy of Sciences, 100012, Beijing, PR China \\ Received 19 February 2002 / Accepted 30 April 2002
}

\begin{abstract}
Blue compact galaxies are compact objects that are dominated by intense star formation. Most of them have dramatically different properties compared to the Milky Way and many other nearby galaxies. Using the IRAS, H I data, and optical spectra, we wanted to measure the current star formation rates, stellar components, metallicities, and star formation histories and evolution of a large blue compact galaxy sample. We anticipate that our study will be useful as a benchmark for studies of emission line galaxies at high redshift. In the first paper of this series, we describe the selection, spectroscopic observation, data reduction and calibration, and spectrophotometric accuracy of a sample of 97 luminous blue compact galaxies. We present a spectrophotometric atlas of rest-frame spectra, as well as tables of the recession velocities and the signal-to-noise ratios. The recession velocities of these galaxies are measured with an accuracy of $\delta V<67 \mathrm{~km} \mathrm{~s}^{-1}$. The average signal-to-noise ratio of sample spectra is $\sim 51$. The spectral line strengths, equivalent widths and continuum fluxes are also measured for the same galaxies and will be analyzed in the next paper of this series. The atlas and tables of measurements will be made available electronically.
\end{abstract}

Key words. atlases - galaxies: active - galaxies: evolution - galaxies: stellar content - techniques: spectroscopic

\section{Introduction}

Blue compact galaxies (BCGs) have luminosities in the range $M_{B} \simeq-12 \mathrm{mag}$ to $M_{B} \simeq-21 \mathrm{mag}$ (Kunth \& Östlin 2000). Much work has been devoted to the study of BCGs, including image (such as Kunth et al. 1988; Doublier et al. 1999; Cairós et al. 2001), and spectra (such as Kunth \& Sargent 1979; Terlevich et al. 1991; Kinney et al. 1993; Kunth et al. 1997) observation in all wavelengths. These galaxies have an average surface brightness higher than $20 \mathrm{mag} \operatorname{arcsec}^{-2}$ (Gordon \& Gottesman 1981), and are characterized by their compact morphology and blue rest-frame colors $(B-V<0.45, B-r<0.6$; Pisano et al. 2001). Most of BCGs have a saturated region surrounded by no, or only small amounts of, nebulosity, and show no regular structures, such as spiral arms, but may display irregular features, such as jets, filaments, bridges, etc.(Gordon \& Gottesman 1981). BCGs were first observed spectroscopically by Sargent \& Searle (1970). Their

Send offprint requests to: X. Kong, e-mail: xkong@mpa-garching.mpg.de

* Table 3 and Fig. 4 are only available in electronic form at the CDS via anonymous ftp to cdsarc.u-strasbg.fr (130.79.128.5) or via

http://cdsweb.u-strasbg.fr/cgi-bin/qcat?J/A+A/389/845 optical spectra show strong narrow emission lines superposed on an almost featureless continuum, similar to the spectrum of an $\mathrm{H}$ II region. The least blue systems generally show both emission and absorption lines, while the bluest usually show only emission lines. The blue rest-frame colors and strong narrow emission line spectra indicate intense current star formation activity (Cairós et al. 2001). Population synthesis models yield typical star formation rates between 1 and $20 M_{\odot} \mathrm{yr}^{-1}$ (Mas-Hesse \& Kunth 1999; Östlin et al. 2001).

Neutral hydrogen observations, in the 21-cm line, of BCGs have shown that BCGs are typically rich in $\mathrm{HI}$ gas, and there are indications that they have large $\mathrm{HI}$ halos (Thuan et al. 1999; Pisano et al. 2001). Another interesting property found among BCGs is that some of them have significant under-abundance of elements heavier than helium. The abundances of heavy elements in these galaxies range between $Z_{\odot} / 50$ and $Z_{\odot} / 2$, and put them among the least chemically evolved galaxies in the universe. The two most metal-deficient galaxies known, I Zw $18\left(Z_{\odot} / 50\right)$ and SBS 0335-052 $\left(Z_{\odot} / 41\right)$ are BCGs (Doublier et al. 1999; Izotov \& Thuan 1999). These properties make BCGs represent an extreme environment for star formation, that differs from that in the Milky Way and in many other nearby galaxies. Detailed studies of these galaxies are not only important for 
understanding their intrinsic properties, but also crucial for understanding star formation processes, galaxy evolution and cosmological parameters (Kunth \& Östlin 2000; Izotov et al. 2001).

The Hubble Space Telescope and the new generation of $8 \mathrm{~m}$ class telescopes have extended our knowledge of the early Universe by identifying galaxies down to magnitudes $B \sim 28 \mathrm{mag}$ and redshifts $z \geq 3$. Studies of intermediateredshift galaxies have revealed a population of compact, luminous galaxies with high star formation rates. Jangren et al. (2002) have compared several of the brighter objects of some intermediate-redshift samples and found that most of the galaxies have small sizes, high luminosities (hence high surface-brightness), and very blue colors, similar to the BCGs (Pisano et al. 2001). Therefore, the more accessible nearby BCGs should help us better understand these distant objects.

Despite the extensive work carried out during the past decade, age and star formation histories of BCGs are not very constrained. With the advent of modern detectors, many BCGs (>90\%) have been found to contain an underlying old stellar population (Kunth \& Östlin 2000, 2001). It suggests that most BCGs undergo a few or several short bursts of star formation followed by longer, more quiescent periods (Kong \& Cheng 1999). An important question is whether these BCGs are unique objects or not? Only a large spectral sample can help us answer this question. The data published so far are not sufficient to allow a systematic analysis of the star formation history of BCGs, because detailed and exhaustive studies have concentrated on particular type of BCGs, such as very metal-deficient galaxies.

Motivated by these facts, we have undertaken an extensive study of a large sample of BCGs, including the collction of IRAS, H I and optical spectroscopy data. Our ultimate goal is to perform a detailed analysis of these galaxies, focusing on the aspects of the various stellar components, the star formation rates, the metallicities (chemical abundances), the age of the underlying population, dust contents and star formation history.

In this paper, we present the blue compact galaxies sample, the optical spectroscopic observations and the method of reduction. The paper is organized as follows: in Sect. 2, we present the galaxy sample. In Sect. 3, we describe the spectral observations. The data reduction, and calibration are outlined in Sect. 4. The spectrophotometric atlas is presented in Sect. 5. In Sect. 6, we assess the data quality and spectrophotometric errors. Finally, some concluding remarks are given in Sect. 7 . In the forthcoming papers of this series, we will study the constraints set by these observations on the physical properties of BCGs in our sample.

\section{Description of the sample}

BCGs are typically rich in $\mathrm{HI}$ gas. Many large $\mathrm{HI}$ surveys of BCGs were carried out, in particular by Gordon \& Gottesman (1981), Thuan \& Martin (1981),
Thuan et al. (1999) and Smoker et al. (2000). Since we want to combine the optical spectra we obtain with HI data to constrain simultaneously the stars and gas contents of BCGs, we therefore select our sample on the basis of these surveys.

Considering the observatory site, instruments and possible observation times, we selected our sample according to the following criteria: (1) $m_{B}^{\mathrm{c}}<17.0 \mathrm{mag}$; (2) $\delta_{2000}>-12^{\circ} ;(3) M_{B}^{c}<-17$ mag. Using these criteria, 83 BCGs were selected. 14 additional "dwarf" $\left(M_{B}^{\mathrm{c}}>-17 \mathrm{mag}\right)$ BCGs were also included because we want to compare their properties with those of "luminous" $\left(M_{B}^{\mathrm{c}}<-17 \mathrm{mag}\right)$ BCGs. The final sample consists of 97 BCGs. We emphasize that there is no guarantee that our sample has any statistical completeness.

Most (92/97) of the galaxies in our sample come from Gordon \& Gottesman (1981). These authors assembled a list of 99 blue compact galaxies from the Markarian, Haro and Zwicky lists. The H I observations were made using the 91-m transit radio telescope of the National Radio Astronomy Observatory and the 305-m spherical radio telescope of the Arecibo Observatory. Three other galaxies in our sample come from Kinney et al. (1993). One galaxy comes from Thuan \& Martin (1981); these authors assembled a list of 115 blue compact dwarf galaxies (BCDs, $\left.M_{B}>-18 \mathrm{mag}\right)$ known at that time from the objective prism surveys of Markarian and Haro, with a few objects from Zwicky and other investigators (we are presently observing most of these BCDs and will present our results in the future). Finally, one galaxy in our sample comes from Thuan et al. (1999).

In total our sample contains 97 blue compact galaxies, the general parameters of which are listed in Table 1. Column 1 lists the galaxy name following Haro (Haro 1956), Markarian (Mrk, Markarian et al. 1989), and Zwicky (Zw, Zwicky 1971). Columns 2 and 3 list the right ascension and declination at epoch 2000 , taken from $\mathrm{NED}^{1}$. Column 4 lists the apparent blue magnitude $\left(m_{B}^{\mathrm{c}}\right)$ corrected for instrumental effects, extinction, inclination, and doppler velocity. Most magnitudes were taken from Gordon \& Gottesman (1981). The other galaxies were corrected for instrumental effects, extinction, inclination based on de Vaucouleurs et al. (1991, RC3) and NED, as indicated by " $\mathrm{R}$ ", "N". Column 5 lists the distance $(D)$ to the galaxy, in megaparsecs, assuming a Hubble constant of $H_{0}=75 \mathrm{~km} \mathrm{~s}^{-1} \mathrm{Mpc}^{-1}$ and $q_{0}=0.5$. Column 6 lists the absolute blue magnitude $\left(M_{B}^{\mathrm{c}},\right)$, derived from Col. 4 and Col. 5 with $M_{B}^{\mathrm{c}}=m_{B}^{\mathrm{c}}-5 \log D+5$. Column 7 lists the Galactic foreground reddening, $E(B-V)$. The last Col. 8 lists other commonly used designations for the galaxies.

The spatial distribution of the 97 sample galaxies on the sky is show in Fig. 1. The absolute blue

1 The NASA/IPAC Extragalactic Database (NED) is operated by the Jet Propulsion Laboratory, California Institute of Technology, under contract with the National Aeronautics and Space Administration. 
Table 1. Global parameters of observed blue compact galaxies.

\begin{tabular}{|c|c|c|c|c|c|c|c|}
\hline $\begin{array}{l}\text { Galaxy } \\
\text { Name }\end{array}$ & $\begin{array}{c}\mathrm{RA}(2000) \\
(\mathrm{hms})\end{array}$ & $\begin{array}{c}\operatorname{Dec}(2000) \\
(\mathrm{dms})\end{array}$ & $\begin{array}{l}m_{B}^{\mathrm{c}} \\
(\mathrm{mag})\end{array}$ & $\begin{array}{r}\text { Dist. } \\
(\mathrm{Mpc})\end{array}$ & $\begin{array}{r}M_{B}^{\mathrm{c}} \\
(\mathrm{mag})\end{array}$ & $\overline{E E(B-V)_{\mathrm{G}}}$ & $\begin{array}{l}\text { Other } \\
\text { Name }\end{array}$ \\
\hline Mrk 335 & $00: 06: 19.5$ & $+20: 12: 10$ & 13.72 & 102.0 & -21.3 & 0.035 & PGC 473 \\
\hline IIIZw12 & $00: 47: 56.5$ & $+22: 22: 23$ & 14.58 & 80.0 & -19.9 & 0.045 & Mrk 347 \\
\hline Haro15 & $00: 48: 35.4$ & $-12: 42: 59$ & 13.48 & 86.0 & -21.2 & 0.023 & Mrk 960 \\
\hline Mrk 352 & $00: 59: 53.3$ & $+31: 49: 37$ & 14.24 & 62.0 & -19.7 & 0.061 & PGC 3575 \\
\hline Mrk 1 & $01: 16: 07.2$ & $+33: 05: 22$ & 14.69 & 67.0 & -19.4 & 0.060 & NGC 449 \\
\hline IIIZw33 & $01: 43: 56.5$ & $+17: 03: 43$ & 14.56 & 109.0 & -20.6 & 0.068 & Mrk 360 \\
\hline VZw155 & $01: 57: 49.4$ & $+27: 51: 56$ & 15.00 & 111.0 & -20.2 & 0.082 & Mrk 364 \\
\hline IIIZw42 & $02: 11: 33.5$ & $+13: 55: 02$ & 14.51 & 107.0 & -20.6 & 0.088 & Mrk 366 \\
\hline IIIZw43 & $02: 13: 45.0$ & $+04: 06: 07$ & 14.05 & 47.0 & -19.3 & 0.042 & Mrk 589 \\
\hline VZw372 & $04: 13: 56.0$ & $+29: 09: 28$ & $15.19 \mathrm{R}$ & 74.0 & -19.2 & 0.709 & UGC 2989 \\
\hline IIZw18 & $04: 38: 39.7$ & $+11: 14: 28$ & $16.41 \mathrm{R}$ & 59.0 & -17.4 & 0.391 & PGC 15715 \\
\hline IIZw23 & $04: 49: 44.4$ & $+03: 20: 03$ & 13.88 & 110.0 & -21.3 & 0.063 & Mrk 1087 \\
\hline IIZw28 & 05:01:42.0 & $+03: 34: 28$ & 14.94 & 113.0 & -20.3 & 0.060 & VV790B \\
\hline IIZw33 & $05: 10: 48.1$ & $-02: 40: 54$ & 13.48 & 36.0 & -19.3 & 0.102 & Mrk 1094 \\
\hline IIZw35 & $05: 16: 59.3$ & $+00: 55: 20$ & $16.90 \mathrm{~N}$ & 95.0 & -18.0 & 0.140 & PGC 17037 \\
\hline IIZw40 & $05: 55: 42.8$ & $+03: 23: 30$ & 14.22 & 9.0 & -15.6 & 0.820 & UGCA 116 \\
\hline IIZw42 & 06:03:11.4 & $+07: 49: 37$ & 15.37 & 69.0 & -18.8 & 0.417 & UGC3 393 \\
\hline Mrk 5 & $06: 42: 15.5$ & $+75: 37: 33$ & 15.12 & 13.3 & -15.5 & 0.084 & UGCA 130 \\
\hline Mrk 6 & $06: 52: 12.2$ & $+74: 25: 37$ & 13.86 & 76.0 & -20.5 & 0.136 & UGC 3547 \\
\hline VIIZw153 & $07: 28: 12.0$ & $+72: 34: 29$ & 13.40 & 43.0 & -19.8 & 0.030 & Mrk 7 \\
\hline VIIZw156 & $07: 29: 25.4$ & $+72: 07: 44$ & 13.17 & 50.0 & -20.3 & 0.026 & Mrk 8 \\
\hline Haro1 & $07: 36: 56.4$ & $+35: 14: 31$ & 12.39 & 50.0 & -21.1 & 0.043 & NGC 2415 \\
\hline Mrk 385 & $08: 03: 28.0$ & $+25: 06: 10$ & 14.57 & 110.0 & -20.6 & 0.030 & PGC 22615 \\
\hline Mrk 622 & $08: 07: 41.0$ & $+39: 00: 15$ & 14.33 & 94.0 & -20.5 & 0.051 & UGC 4229 \\
\hline Mrk 390 & 08:35:33.1 & $+30: 32: 03$ & 14.58 & 101.0 & -20.4 & 0.041 & PGC 24127 \\
\hline Zw0855 & $08: 58: 27.4$ & $+06: 19: 41$ & 14.50 & 47.0 & -18.9 & 0.069 & UGC 4703 \\
\hline Mrk 105 & $09: 20: 26.3$ & $+71: 24: 16$ & 15.88 & 49.0 & -17.6 & 0.066 & PGC 26416 \\
\hline IZw18 & 09:34:02.0 & $+55: 14: 28$ & 15.80 & 10.9 & -14.4 & 0.032 & Mrk 116 \\
\hline Mrk 402 & $09: 35: 19.2$ & $+30: 24: 31$ & $15.66 \mathrm{R}$ & 98.0 & -19.3 & 0.020 & PGC 27258 \\
\hline IZw21 & $09: 46: 28.6$ & $+45: 45: 09$ & 14.59 & 67.0 & -19.5 & 0.015 & UGC 5225 \\
\hline Haro22 & $09: 50: 11.0$ & $+28: 00: 47$ & 14.94 & 18.4 & -16.4 & 0.025 & PGC 28305 \\
\hline Haro23 & 10:06:18.1 & $+28: 56: 40$ & 14.33 & 17.5 & -16.9 & 0.024 & UGCA 201 \\
\hline IIZw44 & $10: 15: 14.7$ & $+21: 06: 34$ & 15.75 & 81.0 & -18.8 & 0.023 & PGC 29934 \\
\hline Haro2 & $10: 32: 31.9$ & $+54: 24: 03$ & 13.15 & 21.0 & -18.5 & 0.012 & Mrk 33 \\
\hline Mrk 148 & $10: 35: 34.8$ & $+44: 18: 57$ & $14.65 \mathrm{R}$ & 96.0 & -20.3 & 0.019 & UGC 5747 \\
\hline Haro3 & $10: 45: 22.4$ & $+55: 57: 37$ & 12.81 & 13.6 & -17.9 & 0.007 & Mrk 35 \\
\hline Haro25 & $10: 48: 44.2$ & $+26: 03: 12$ & 15.52 & 101.0 & -19.5 & 0.033 & Mrk 727 \\
\hline Mrk 1267 & $10: 53: 03.9$ & $+04: 37: 54$ & 14.48 & 77.8 & -20.0 & 0.034 & Ark $264^{r 1}$ \\
\hline Haro4 & 11:04:58.5 & $+29: 08: 22$ & 15.51 & 7.9 & -14.0 & 0.030 & Mrk 36 \\
\hline $\mathrm{IZw} 26$ & $11: 25: 36.2$ & $+54: 22: 57$ & 16.80 & 83.0 & -17.8 & 0.014 & Mrk 40 \\
\hline Mrk169 & $11: 26: 44.4$ & $+59: 09: 20$ & 13.95 & 17.5 & -17.3 & 0.014 & UGC 6447 \\
\hline Haro27 & $11: 40: 24.8$ & $+28: 22: 26$ & 14.13 & 24.0 & -17.8 & 0.022 & Mrk 1507 \\
\hline Mrk198 & 12:09:14.1 & $+47: 03: 30$ & 14.56 & 97.0 & -20.4 & 0.017 & PGC 38613 \\
\hline IIZw57 & $12: 09: 32.9$ & $+17: 00: 51$ & 13.60 & 88.0 & -21.1 & 0.036 & PGC 38634 \\
\hline Mrk201 & $12: 14: 09.7$ & $+54: 31: 38$ & 12.86 & 35.0 & -19.9 & 0.015 & $\mathrm{IZw} 33^{r 1}$ \\
\hline Haro28 & $12: 15: 46.1$ & $+48: 07: 54$ & 13.21 & 10.6 & -16.9 & 0.016 & NGC 4218 \\
\hline Haro8 & $12: 19: 09.9$ & $+03: 51: 21$ & 14.49 & 18.6 & -16.9 & 0.017 & Mrk 49 \\
\hline Mrk50 & $12: 23: 24.1$ & $+02: 40: 45$ & $14.89 \mathrm{R}$ & 92.0 & -19.9 & 0.016 & PGC 40220 \\
\hline Haro29 & $12: 26: 16.0$ & $+48: 29: 37$ & 14.24 & 4.8 & -14.2 & 0.015 & IZw 36 \\
\hline
\end{tabular}

magnitude $\left(M_{B}^{\mathrm{c}}\right)$ distribution of the 97 sample galaxies is presented in Fig. 2.

\section{Spectroscopic observations}

Two-dimensional (long-slit) spectra were obtained using the OMR (Optomechanics Research Inc.) spectrograph mounted at the Cassegrain focus of the $2.16 \mathrm{~m}$ telescope at the XingLong Station of the Beijing Astronomical
Observatory (BAO) in China, which also belongs to the National Astronomical Observatories, Chinese Academy of Sciences. The data were acquired during 26 predominantly moonless nights between March 1997 and January 2002. The vast majority of the observations were obtained during transparent or photometric conditions, the remainder through thin clouds. 
Table 1. continued.

\begin{tabular}{|c|c|c|c|c|c|c|c|}
\hline $\begin{array}{l}\text { Galaxy } \\
\text { Name }\end{array}$ & $\begin{array}{l}\text { RA (2000) } \\
(\mathrm{hms})\end{array}$ & $\begin{array}{c}\text { Dec. }(2000) \\
(\mathrm{dms})\end{array}$ & $\begin{array}{l}m_{B}^{\mathrm{c}} \\
(\mathrm{mag})\end{array}$ & $\begin{array}{r}\text { Dist. } \\
(\mathrm{Mpc})\end{array}$ & $\begin{array}{r}M_{B}^{\mathrm{c}} \\
(\mathrm{mag})\end{array}$ & $E(B-V)_{\mathrm{G}}$ & $\begin{array}{l}\text { Other } \\
\text { Name }\end{array}$ \\
\hline Mrk 213 & $12: 31: 22.2$ & $+57: 57: 52$ & $13.03 \mathrm{R}$ & 41.7 & -20.1 & 0.012 & NGC $4500^{r 1}$ \\
\hline Mrk 215 & $12: 32: 34.7$ & $+45: 46: 04$ & 14.47 & 79.0 & -20.0 & 0.017 & PGC 41591 \\
\hline Haro32 & $12: 43: 48.6$ & $+54: 54: 02$ & 13.70 & 67.0 & -20.4 & 0.016 & $\mathrm{IZw} 41$ \\
\hline Haro33 & $12: 44: 38.3$ & $+28: 28: 19$ & 14.39 & 12.5 & -16.1 & 0.016 & UGCA 294 \\
\hline Haro34 & $12: 45: 06.6$ & $+21: 10: 10$ & 14.86 & 93.0 & -20.0 & 0.041 & Ark 386 \\
\hline Haro36 & $12: 46: 56.4$ & $+51: 36: 46$ & 14.67 & 8.0 & -14.8 & 0.015 & UGC 7950 \\
\hline Haro35 & $12: 47: 08.5$ & $+27: 47: 35$ & $15.89 \mathrm{R}$ & 99.0 & -19.1 & 0.013 & PGC 43139 \\
\hline Haro37 & $12: 48: 41.0$ & $+34: 28: 39$ & 15.18 & 57.0 & -18.6 & 0.014 & Mrk 444 \\
\hline IIIZw68 & $12: 58: 02.4$ & $+26: 51: 34$ & 14.34 & 76.0 & -20.1 & 0.009 & UGC 8080 \\
\hline IIZw67 & $12: 58: 35.2$ & $+27: 35: 47$ & 13.69 & 101.0 & -21.3 & 0.010 & NGC 4853 \\
\hline Mrk 57 & $12: 58: 37.2$ & $+27: 10: 34$ & 15.08 & 102.0 & -20.0 & 0.014 & PGC $44486^{r 2}$ \\
\hline Mrk 235 & 13:00:02.1 & $+33: 26: 15$ & 15.07 & 100.0 & -19.9 & 0.012 & PGC 44694 \\
\hline Mrk 241 & $13: 06: 19.8$ & $+32: 58: 25$ & 15.87 & 105.0 & -19.2 & 0.014 & PGC 45363 \\
\hline IZw53 & $13: 13: 57.7$ & $+35: 18: 55$ & $15.00 \mathrm{R}$ & 69.0 & -19.2 & 0.011 & PGC 45993 \\
\hline IZw56 & $13: 20: 35.3$ & $+34: 08: 22$ & 14.10 & 93.0 & -20.7 & 0.013 & UGC 8387 \\
\hline Haro38 & $13: 35: 35.6$ & $+29: 13: 01$ & 14.87 & 11.9 & -15.5 & 0.012 & UGC 8578 \\
\hline Mrk 270 & $13: 41: 05.7$ & $+67: 40: 20$ & 13.61 & 38.0 & -19.3 & 0.020 & NGC 5283 \\
\hline Mrk 275 & $13: 48: 40.5$ & $+31: 27: 39$ & 14.52 & 106.0 & -20.6 & 0.019 & PGC 48992 \\
\hline Haro39 & $13: 58: 23.8$ & $+25: 33: 00$ & 14.49 & 35.0 & -18.2 & 0.016 & PGC 49732 \\
\hline Haro42 & 14:31:09.0 & $+27: 14: 14$ & 14.74 & 60.0 & -19.2 & 0.015 & Mrk 685 \\
\hline Haro43 & $14: 36: 08.8$ & $+28: 26: 59$ & 15.08 & 27.0 & -17.1 & 0.020 & PGC 52193 \\
\hline Haro44 & $14: 43: 24.7$ & $+28: 18: 04$ & $16.05 \mathrm{R}$ & 51.0 & -17.5 & 0.017 & PGC 52587 \\
\hline IIZw70 & $14: 50: 56.5$ & $+35: 34: 18$ & 14.04 & 18.0 & -17.2 & 0.012 & Mrk 829 \\
\hline IIZw71 & $14: 51: 14.4$ & $+35: 32: 31$ & 13.34 & 18.0 & -17.9 & 0.013 & PGC 53039 \\
\hline IZw97 & $14: 54: 39.2$ & $+42: 01: 26$ & 14.47 & 36.0 & -18.3 & 0.020 & PGC 53299 \\
\hline IZw98 & $14: 55: 15.6$ & $+42: 30: 25$ & 13.91 & 75.0 & -20.5 & 0.019 & NGC 5787 \\
\hline IZw101 & $15: 03: 45.8$ & $+42: 41: 59$ & 15.19 & 68.0 & -19.0 & 0.019 & Ark467 \\
\hline IZw117 & $15: 35: 53.6$ & $+38: 40: 37$ & 14.15 & 77.0 & -20.3 & 0.017 & UGC 9922 \\
\hline IZw123 & $15: 37: 04.2$ & $+55: 15: 48$ & 15.44 & 11.6 & -14.9 & 0.014 & Mrk 487 \\
\hline VIIZw631 & $15: 59: 11.9$ & $+20: 45: 31$ & 12.98 & 62.0 & -21.0 & 0.055 & NGC 6027 \\
\hline Mrk 297 & $16: 05: 12.9$ & $+20: 32: 32$ & 12.98 & 65.0 & -21.1 & 0.076 & NGC 6052 \\
\hline IZw147 & $16: 23: 12.0$ & $+54: 08: 36$ & $15.80 \mathrm{R}$ & 75.0 & -18.6 & 0.009 & PGC 57975 \\
\hline IZw159 & $16: 35: 21.1$ & $+52: 12: 53$ & 15.70 & 38.0 & -17.2 & 0.029 & Mrk 1499 \\
\hline IZw166 & $16: 48: 24.1$ & $+48: 42: 33$ & 15.03 & 106.0 & -20.1 & 0.016 & Mrk 499 \\
\hline Mrk 893 & $17: 15: 02.2$ & $+60: 12: 59$ & 16.34 & 83.7 & -18.3 & 0.021 & $1714+602^{r 3}$ \\
\hline IZw191 & $17: 40: 24.8$ & $+47: 43: 59$ & $14.70 \mathrm{R}$ & 81.0 & -19.8 & 0.019 & PGC 60671 \\
\hline IZw199 & $17: 50: 05.1$ & $+56: 40: 27$ & 14.82 & 74.0 & -19.5 & 0.048 & PGC 60950 \\
\hline IZw207 & $18: 31: 10.4$ & $+55: 16: 32$ & $15.31 \mathrm{R}$ & 79.0 & -19.2 & 0.042 & PGC 61982 \\
\hline IIZw82 & $20: 23: 15.6$ & $+00: 39: 52$ & 13.46 & 56.0 & -20.3 & 0.191 & UGC 11546 \\
\hline IVZw67 & 21:02:18.8 & $+36: 41: 44$ & $14.00 \mathrm{~N}$ & 38.0 & -18.9 & 0.280 & UGC 11668 \\
\hline IIZw172 & $22: 14: 45.9$ & $+13: 50: 37$ & 13.23 & 108.0 & -21.9 & 0.065 & NGC 7237 \\
\hline IVZw93 & $22: 16: 07.7$ & $+22: 56: 33$ & $15.44 \mathrm{R}$ & 55.0 & -18.3 & 0.069 & PGC 68454 \\
\hline Mrk 303 & $22: 16: 26.8$ & $+16: 28: 17$ & 14.62 & 104.0 & -20.5 & 0.051 & NGC 7244 \\
\hline Zw2220 & $22: 23: 02.0$ & $+30: 55: 29$ & 13.52 & 93.0 & -21.3 & 0.078 & UGC 12011 \\
\hline Mrk 314 & $23: 02: 59.2$ & $+16: 36: 19$ & 13.45 & 31.0 & -19.0 & 0.085 & Mrk 314 \\
\hline IVZw142 & $23: 20: 03.1$ & $+26: 12: 58$ & 14.89 & 109.0 & -20.3 & 0.086 & Mrk 322 \\
\hline IVZw149 & $23: 27: 41.2$ & $+23: 35: 21$ & 12.66 & 80.0 & -21.9 & 0.043 & Mrk 325 \\
\hline Zw2335 & $23: 37: 39.6$ & $+30: 07: 47$ & 15.37 & 22.0 & -16.3 & 0.093 & Mrk 328 \\
\hline
\end{tabular}

r1: Kinney et al. (1993); r2: Thuan \& Martin (1981); r3: Thuan et al. (1999);

The other BCGs were selected from Gordon \& Gottesman (1981). 


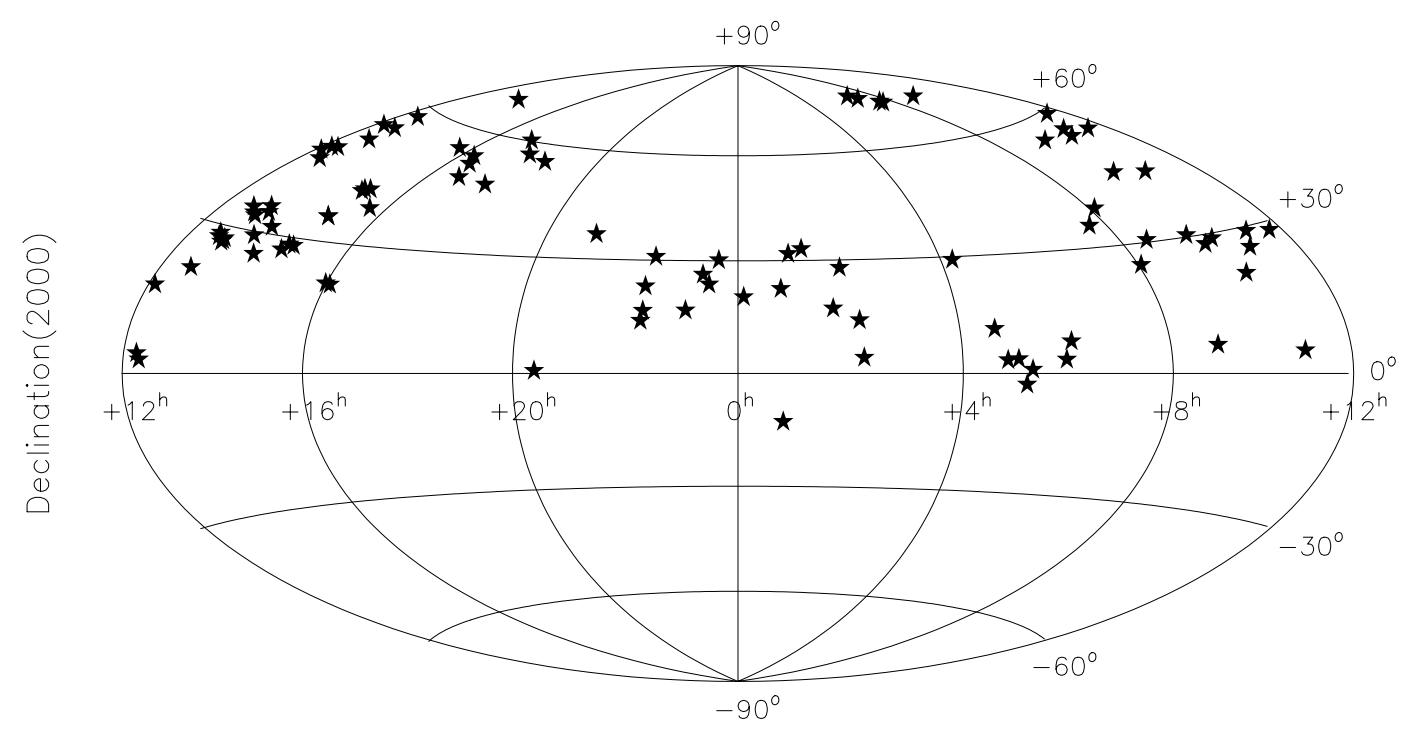

Right Ascension(2000)

Fig. 1. The distribution of blue compact galaxies in the sky shown in equal-area projection, centered on Declination (latitude) 0, Right Ascension (longitude) 0.

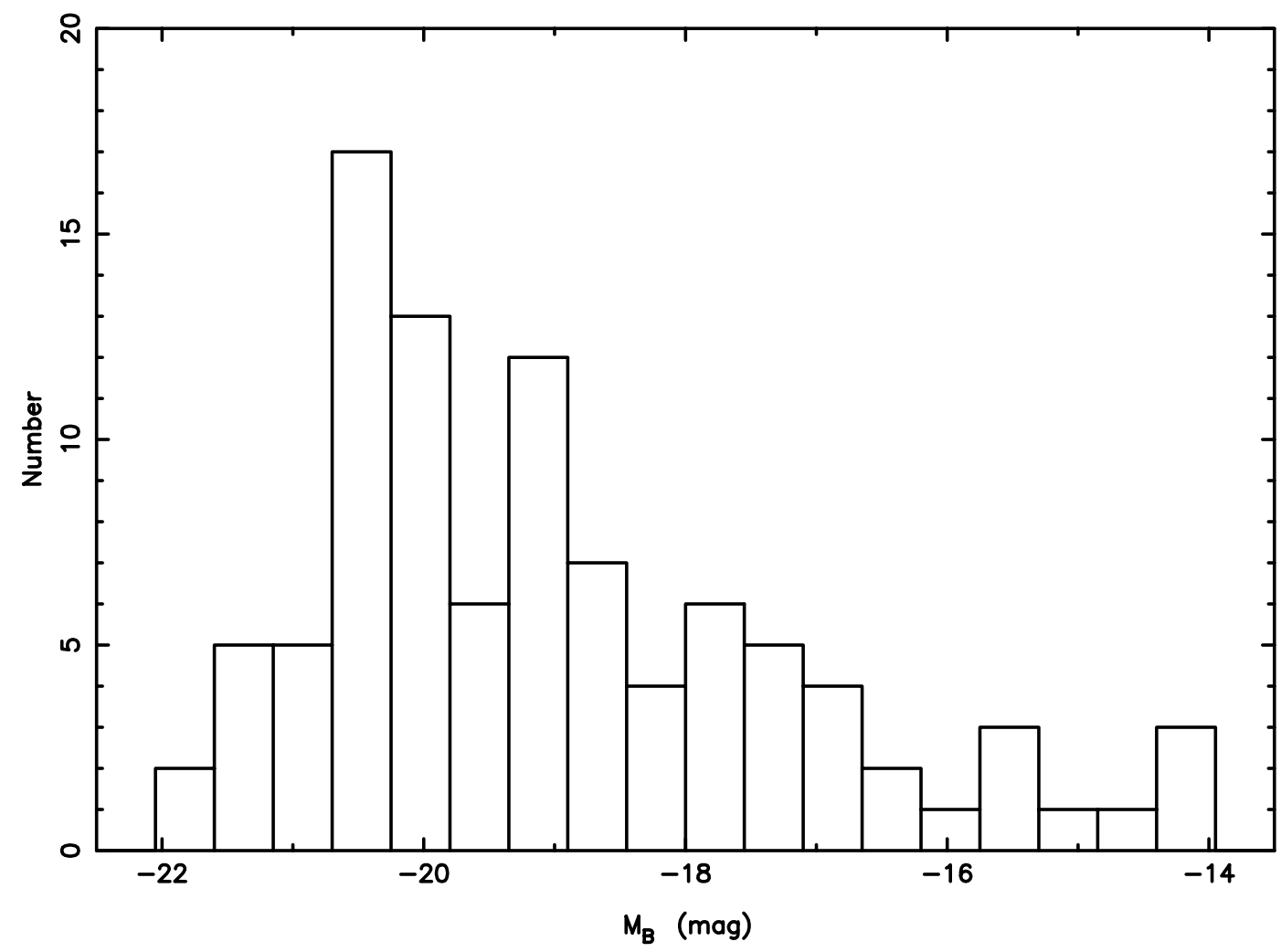

Fig. 2. The distribution of absolute blue magnitudes $\left(M_{B}\right)$ of the 97 blue compact galaxies in our sample, 83 have $M_{B}<-17$ mag.

The OMR spectrograph was equipped with a TEKTRONIX TEK1024, AR-coated back-illuminated CCD with $1024 \times 1024,24 \mu \mathrm{m}$ pixels. A 300 line $\mathrm{mm}^{-1}$ grating blazed at $5500 \AA$ in first order was used, giving a dispersion of $4.8 \AA$ pixel $^{-1}$ and a total spectral coverage of $\sim 4500 \AA$. According to the condition of seeing, we adjusted the slit width in between $2^{\prime \prime}$ and $3^{\prime \prime}$ on each night and used the selected configuration for all target spectra. Our spectra were centered on $5500 \AA$ or $6000 \AA$ (see Table 2 ), at a resolution $(F W H M)$ of $\sim 10 \AA$, thus 
Table 2. The observation date and instrumental parameters.

\begin{tabular}{rlccccrl}
\hline \hline $\begin{array}{c}\text { Obs. } \\
\text { No. }\end{array}$ & Observation date & $\begin{array}{c}\text { Number } \\
\text { of nights }\end{array}$ & $\begin{array}{c}\text { cw. } \\
(\AA)\end{array}$ & $\begin{array}{l}\text { slit } \\
\left({ }^{\prime \prime}\right)\end{array}$ & $\begin{array}{c}\text { arc } \\
\text { lamp }\end{array}$ & $\begin{array}{r}\text { Spec. } \\
\text { num. }\end{array}$ & Standard stars \\
\hline 1 & 2002 Jan. 08-Jan. 12 & 5 & 5500 & 2.5 & $\mathrm{He} / \mathrm{Ar}$ & 20 & G191B2B Hilt600 Feige56 Feige25 HZ44 \\
2 & 2001 Oct. 12 & 1 & 5500 & 2.5 & $\mathrm{He} / \mathrm{Ar}$ & 4 & Hilt600 HD192281 G191B2B BD174708 \\
3 & 2001 Feb. 24-Mar. 01 & 4 & 5500 & 2.5 & $\mathrm{He} / \mathrm{Ar}$ & 33 & G191B2B Feige34 BD332642 HZ44 \\
4 & 2000 Apr. 29-May. 01 & 3 & 6000 & 2.3 & $\mathrm{He} / \mathrm{Ar}$ & 24 & Feige34 BD332642 HZ44 \\
5 & 2000 Feb. 25 & 1 & 6000 & 2.2 & $\mathrm{He} / \mathrm{Ar}$ & 4 & Hilt600 Feige34 \\
6 & 2000 Jan. 01-Jan. 02 & 2 & 5500 & 3.0 & $\mathrm{He} / \mathrm{Ar}$ & 17 & G191B2B Hilt600 Feige34 \\
7 & 1999 Aug. 03-Aug. 09 & 2 & 5500 & 2.0 & $\mathrm{He} / \mathrm{Ar}$ & 4 & BD284211 HD192281 \\
8 & 1998 Aug. 14-Aug. 20 & 4 & 5500 & 2.5 & $\mathrm{He} / \mathrm{Ar}$ & 15 & BD284211 Kopff27 Feige15 BD332642 \\
9 & 1998 Feb. 17 & 1 & 5500 & 2.0 & $\mathrm{He} / \mathrm{Ar}$ & 2 & Hilt600 Feige98 \\
10 & 1997 Mar. 18-Mar. 20 & 3 & 6000 & 2.0 & $\mathrm{Fe} / \mathrm{Ar}$ & 13 & Feige34 Feige98 Sa29130 \\
\hline
\end{tabular}

covering many interesting spectral features ranging from $[\mathrm{O}$ II $] \lambda 3727$ in the blue to $[\mathrm{S}$ II $] \lambda 6731$ in the red.

The slit was generally placed across the nucleus of each galaxy. In some cases where the location of the nucleus was not obvious, the slit was aligned to intersect the brightest part of the galaxy in order to maximize the chance of detecting the nucleus. Otherwise, the spectrograph was rotated about the optical axis of the telescope as required to keep the slit approximately perpendicular to the horizon, to minimize loss of light due to differential atmospheric refraction (e.g., Filippenko 1982).

Dome flats, illuminated by a hot, spectroscopically featureless arc lamp, were taken at the beginning and end of each night. He-Ar or Fe-Ar (before 1998, see Col. 6 of Table 2) lamps were observed immediately after observing each target object at the same position as the object for wavelength calibration.

Two or more of the KPNO spectrophotometric standards from Massey et al. (1988), such as G191B2B, Hiltner 600, Feige 34, HZ 44, BD +28 4211, were observed each night for absolute photometric calibration. In good conditions, a standard was observed about every 3 or 4 hours.

Table 2 lists the observations log and instrumental parameters for each run. Column 1 lists the observation run number, Col. 2 the observation period, and Col. 3 the number of nights actually observed, i.e. that were not lost because of poor weather conditions. Column 4 lists the centering wavelength used for each run. Columns 5-8 list the slit width, the arc lamp used, the number of spectral obtained, and the standard stars for each run. The individual integration times in each of the observations varied from $1200 \mathrm{~s}$ to about an hour, depending on the magnitude of the object and the seeing. For 39 objects, observations were performed twice in order to get a higher signal-to-noise ratio spectrum, so that we have observed a total 136 BCGs spectra. 107 of the total 136 spectra were observed at airmass less than 1.2 , only 4 spectra were observed at airmass larger than 1.5. Table 3 lists these observation parameters. Column 1 lists the object name, while Cols. 2-4 and 5-7 list the observation date, exposure time, and airmass.

\section{Data reduction}

The spectroscopic reductions were made using the Image Reduction Analysis Facility (IRAF) ${ }^{1}$ packages CCDRED, TWODSPEC and ONEDSPEC. For each night of data, the following steps were performed: (a) interpolation over bad columns, dead and hot pixels; (b) bias subtraction; (c) division of each frame by a flat-field exposure to remove multiplicative gain and illumination variations across the chip; (d) extraction of one dimensional spectra for each observation from the two-dimensional image by summing the pixels within the aperture at each point along the dispersion axis and subtracting out the sky background; (e) wavelength calibration and subsequent resampling of the data on a linear wavelength grid; (f) flux calibration of the extracted spectra, using flux standard stars from the KPNO standards sample; (g) correction for extinction using a standard atmospheric extinction law.

\subsection{Basic reductions}

Pixel-to-pixel variations in the response were removed through division by appropriately normalized exposures of the dome illuminated by a hot, spectroscopically featureless lamp. The dark counts were so low that their subtraction was not performed. Cosmic ray events in each input image were detected and replaced by the average of the four neighbors with the IRAF task cosmicrays. The remaining cosmic ray hits were flagged manually and were subsequently removed by interpolation.

We extracted the spectra using objectively defined apertures. The peak of the galaxy light distribution was used to trace the extraction aperture. For the object spectra, we used a fixed aperture, 6 pixels along the slit centered on the brightest pixel in the portion of the spectrum between 5300-5600 A. The background sky level was determined from areas as close to the nucleus as possible, taking care not to include the contribution from extended emission near the nucleus. One-dimensional spectra of the

\footnotetext{
${ }^{1}$ IRAF is distributed by the National Optical Astronomy Observatories, which are operated by the Association of Universities for Research in Astronomy, Inc., under cooperative agreement with the National Science Foundation.
} 
standard stars were extracted in exactly the same manner as for the galaxy spectra, using an effective slit length to contain all the stellar light.

Wavelength calibration was carried out by fitting a cubic spline to unblended emission lines of $\mathrm{He}, \mathrm{Ar}$, and $\mathrm{Fe}$ in the comparison lamp spectra. These spectra were also used to measure the spectral resolution as a function of position on the CCDs. More than 20 lines were used to establish the wavelength scale. Typical rms residuals in the cubic spline fits were $0.3 \AA$. The accuracy of the wavelength calibration was better than $1.5 \AA$.

The extracted spectra were flux calibrated on a relative flux scale using more than two KPNO standard stars. Cubic spline sensitivity functions of ninth order were fit interactively for each of the standard star observations. The sensitivity function relates the measured intensity to the (calibrated) flux density (in $\operatorname{ergss}^{-1} \mathrm{~cm}^{-2} \AA^{-1}$ ) as a function of wavelength, after removing atmospheric extinction.

Atmospheric extinction was corrected using the mean extinction coefficients for the Xinglong station (BAO), that were measured in the BeijingArizona-Taiwan-Connecticut (BATC) multi-color survey (Kong et al. 2000). There is little error introduced by this procedure, since the observations were restricted to small air masses, usually less than 1.2 and always less than 1.7.

The telluric $\mathrm{O}_{2}$ absorption lines near 6280 and $6860 \AA$ (the " $B$ band") were removed through division by normalized, intrinsically featureless spectra of the standard stars. Large residuals caused by mismatches at the sharp, deep band were not removed.

\subsection{Additional reductions}

To measure the rest-frame spectral line properties of the galaxies, we first measured the recession velocity of each galaxy by averaging the recession velocities, $V_{\mathrm{o}}=c \Delta \lambda / \lambda_{0}$ of different lines, where $\lambda_{0}$ is the rest-frame wavelength of the line.

For galaxies with emission lines, recession velocities were determined from the average of five measurable emission lines, [O II] $\lambda 3727, \mathrm{H} \beta \lambda 4861$, [O III] $\lambda 4959$, [O III] $\lambda 5007$, $\mathrm{H} \alpha \lambda 6563$. For those objects without emission lines, velocities were obtained from the average of 5 measurable absorption lines, CaK $\lambda 3935, \mathrm{H} \delta \lambda 4101, G$ band $\lambda 4306$, $\mathrm{Mg} \mathrm{I}+\mathrm{Mg} \mathrm{H} \lambda 5177$ and $\mathrm{Na} \mathrm{I} \lambda 5896$. For the galaxy, IVZw 67 , that has neither strong emission nor absorption lines, we adopted the redshift listed by NED. The accuracy of the recession velocities in our sample ranges from very good (3-55 $\mathrm{km} \mathrm{s}^{-1}$ uncertainties) when the galaxy has strong emission lines, to relatively poor $\left(7-67 \mathrm{~km} \mathrm{~s}^{-1}\right.$ uncertainties) when the galaxy has only absorption lines. The recession velocity distribution for all the sample galaxies with spectroscopic observations from this study are presented in Fig. 3. All galaxies in the sample have velocities below $9000 \mathrm{~km} \mathrm{~s}^{-1}$. On the basis of these recession velocities, the calibrated spectra were shifted to rest-frame wavelengths.
Columns 8-9 of Table 3 list the galaxy recession velocities in $\mathrm{km} \mathrm{s}^{-1}$, the uncertainty of the recession velocity.

The foreground reddening cause by our Galaxy was corrected using the values in Col. 7 of Table 1 . The wavelength dependence of the extinction was assumed to follow the empirical selective extinction function of Cardelli et al. (1989), with $R_{V}=A_{V} / E(B-V)=3.1$.

The rest frame spectra were normalized to the average flux in a $50 \AA$ interval centered at $5500 \AA$ and subsequently resampled on a uniform wavelength grid spanning the range $3580-7600 \AA$.

For each galaxy, multiple exposures taken with the same setting, sometimes over several epochs, were combined in a weighted average, with the weights determined by the signal-to-noise ratios.

\section{The spectral atlas}

The calibrated spectra of the nuclei of all survey galaxies are presented in Fig. 4. The objects are ordered with increasing right ascension at the epoch $2000\left(\alpha_{2000}\right)$, as in Table 1. The spectra have all been normalized to the flux in a $50 \AA$ wavelength interval centered on $5500 \AA$. The ordinate displays normalized flux, $F(\lambda) / F(5500)$, and the abscissa wavelength $(\AA)$ in the restframe of the galaxy, binned linearly to $2 \AA$. All spectra were plotted on a common wavelength scale of $3580-7600 \AA$.

The spectra are generally of high quality. The signalto-noise ratios $(S N R \mathrm{~s})$ of the spectra, measured at $5500 \AA$ over a region between 5475 and $5525 \AA$, are high. The SNRs of 89 sample galaxies are larger than 20, the average over the sample galaxies being $\sim 51$. Columns $10-11$ of Table 3 list the flux and signal-to-noise ratios in a $50 \AA$ wavelength interval centered on $5500 \AA$.

Because the original BCGs sample of Haro (1956), Markarian et al. (1989), and Zwicky (1971) were identified on the basis of their high surface brightness on photographic plates and strong UV emission, they do not all have spectra similar to those of HiI regions. We find that some BCGs have broad $\mathrm{H} \alpha$ spectral lines, such as Mrk 6, Mrk 335, and other objects. In fact, based on Véron-Cetty \& Véron (2001), 10 sample galaxies are found to be Seyfert galaxies. 13 of 97 BCGs have no emission lines. The other 74 spectra are typical H II region spectra, with strong narrow nebular emission lines.

The atlas and tables of measurements will be made available electronically.

\section{Discussion}

\subsection{Spectrophotometric accuracy}

The spectrophotometric accuracy can be estimated by adding individual contributions to the total error in quadrature.

The main sources of errors that affect the spectral shape include: the fitting of the sensitivity function, the published standard star fluxes, the adopted atmospheric 


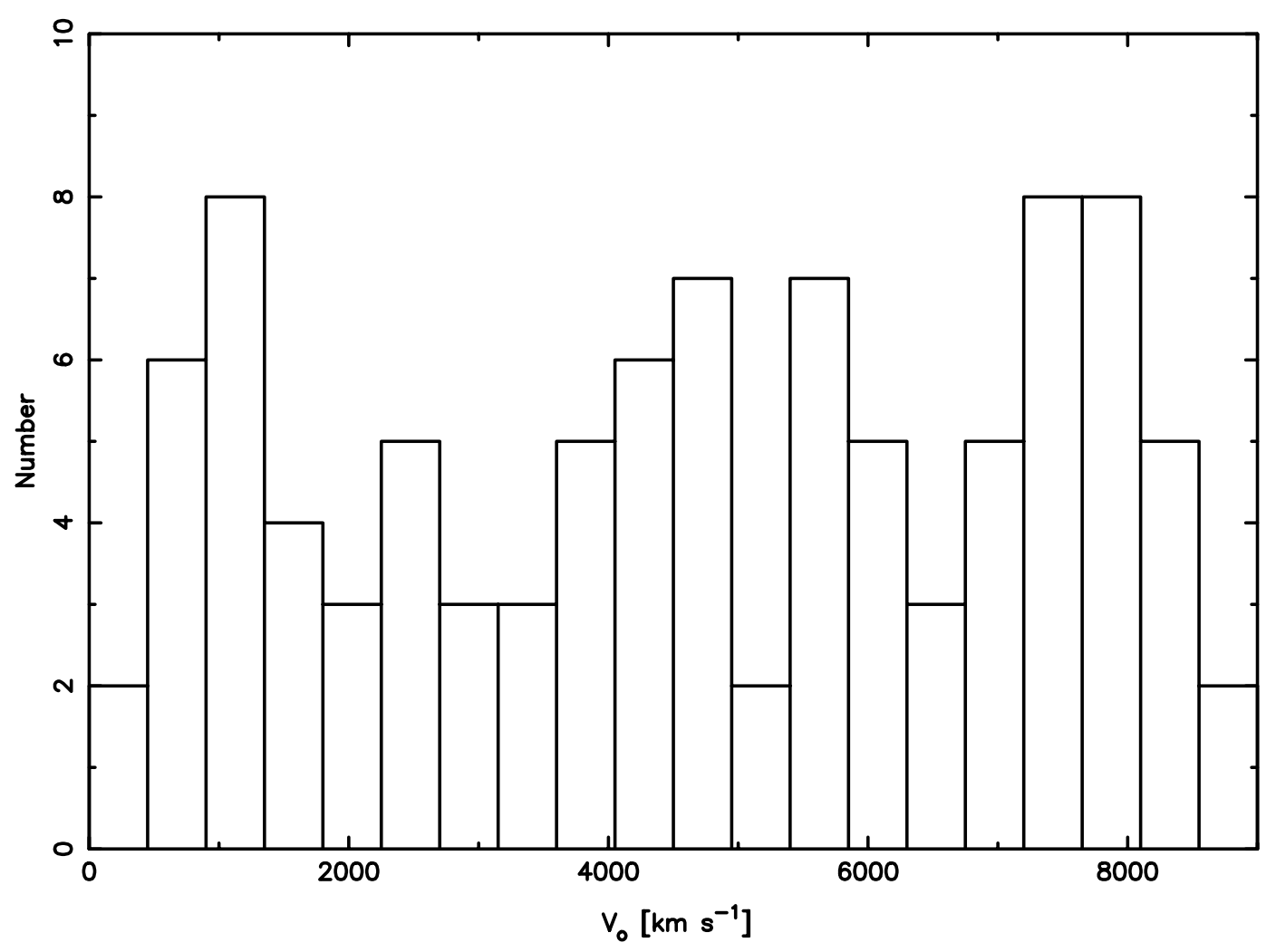

Fig. 3. Histogram of the recession velocity distribution for all the 97 sample galaxies.

extinction curve (Jansen et al. 2000). The error in the fit of the sensitivity function is estimated using the residuals of individual standard stars from the mean calibration. These residuals are dominated by systematic differences in the sensitivity function fitted to the different stars, and are less than 5\%. Standard star fluxes are accurate to better than 3\% (Massey et al. 1988). Considering this 3\% uncertainty, we find that errors in our sensitivity function fits are likely to be less than $5 \%$. Application of the BAO mean atmospheric extinction curve to correct our data introduces an error in the continuum slope of the spectra. Because most galaxies were observed at low airmasses, we expect the error in the continuum slope to be less than $5 \%$ at any wavelength.

The main sources of errors that affect restricted ranges in wavelength include: the flat field variations, wavelength calibration, the sky subtraction and residuals due to cosmic ray hits (Jansen et al. 2000). Differences between flat fields taken on different nights within a run are small. The contribution of the read noise to the error in the flat field is negligible. Errors in the wavelength calibration introduce small errors in the inferred flux densities on scales comparable to the distance between individual calibration lines. Errors in the dispersion solution are less than $0.3 \AA$. These dispersion errors produce spectrophotometric errors of at most 3\%. Sky subtraction errors dominate the total error on small scales. Because BCGs are compact objects, errors from sky subtraction are less than 5\%. Cosmic ray residuals introduce large errors in the extracted spectra only near emission lines, where the steepness of the local background renders a clean fit difficult. Residuals in continuum or sky portions in the spectra are smaller than $2 \%$ of the local background. Errors may be as large as $10 \%$ per extracted pixel.

This analysis shows that the spectrophotometry is accurate to better than $10 \%$ over small wavelength regions, and about $15 \%$ or better on large scales.

\subsection{Internal checks of the spectrophotometry}

In Table 3 we list the total number of observations of each galaxy; 39 galaxies have been observed twice. To check how consistent our calibration procedure is from night to night, including whatever errors exist in the adoption of a mean extinction curve, we can compare the final spectrophotometry to each individual observation.

To illustrate this level of accuracy, we show in Figure 5a. duplicate spectra for three kinds of galaxies: an emission line galaxy (VIIZw153), an absorption line galaxy (IIZw35), and a Seyfert galaxy (Mrk 335). The dates at which the spectra were obtained, the exposure times, and the effective airmasses during the observations are indicated. As expected, the deviations are rarely larger than $10 \%$ from 4500 to $6800 \AA$. We see that the average spectral energy distribution precision of our data is good. No systematic differences are seen.

\subsection{Comparison with other spectra}

Our sample of blue compact galaxies contains two galaxies that were also observed by Kennicutt (1992): Haro 3 and 


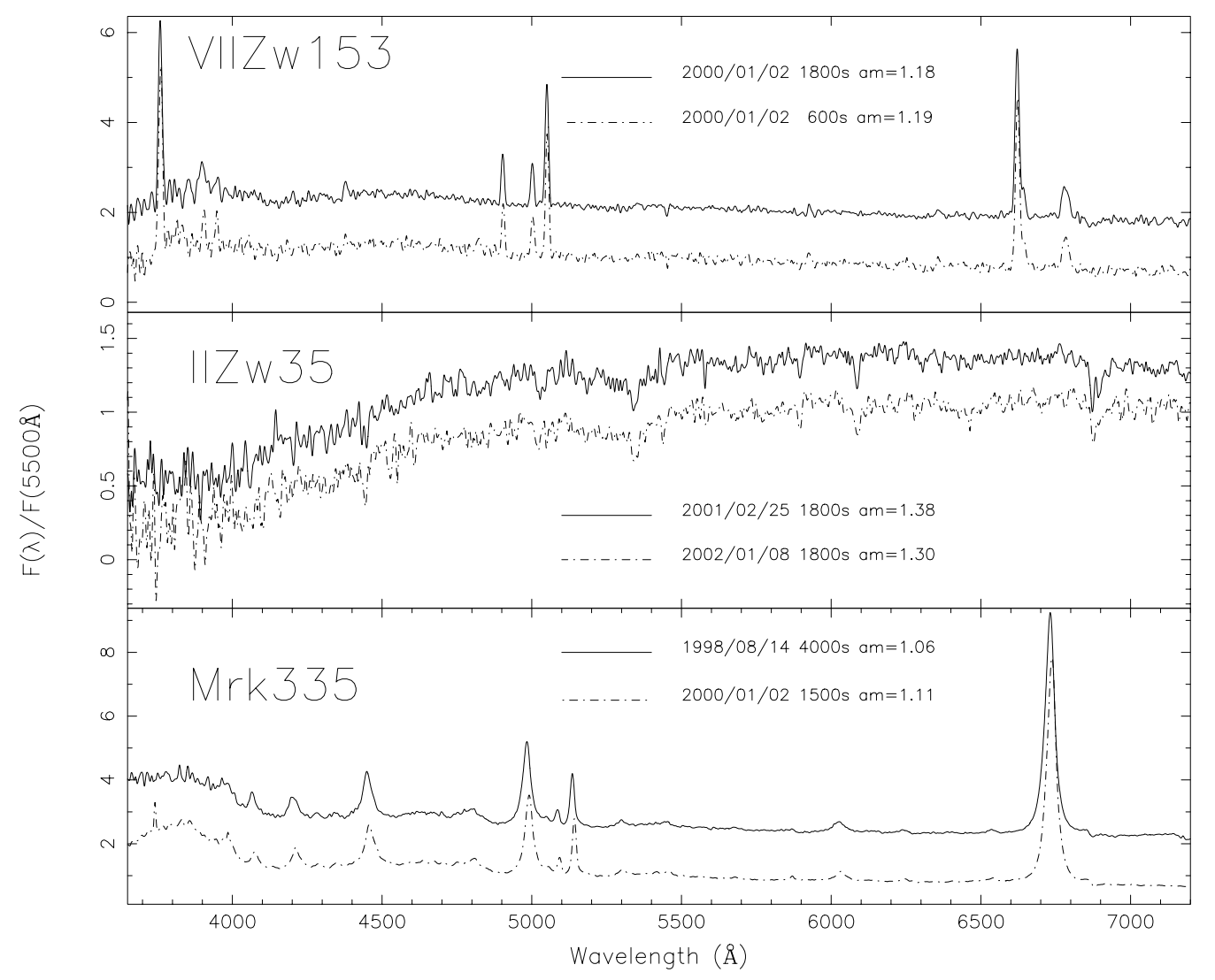

Fig. 5. a) A check of the internal consistency of our spectrophotometry using the spectra of the 3 galaxies that we observed during two different times. VII Zw 153: emission line galaxy; II Zw 35: absorption line galaxy; Mrk 335: Seyfert galaxy. All spectra were normalized to the average level in interval 5475-5525 $\AA$. The spectra in each panel have been offset for clarity.

Mrk 201. We compare our spectra for these objects with those obtained by Kennicutt (1992) in Fig. 5b.

In the $4500-6800 \AA$ region the continuum of our spectra match Kennicutt's to better than $10 \%$ over small ranges. Bluewards of $4500 \AA$ differences tend to become larger, up to about $30 \%$. In addition, our spectra show a bluer optical spectrum and stronger emission lines than do Kennicutt's. The aperture used by Kennicutt is much larger than ours ( $45^{\prime \prime}$ circular versus $3^{\prime \prime}$ slit). Since BCGs have a central burst of star formation, the difference in aperture size is most likely the cause of the difference in continuum shape and emission lines. The increased aperture size allows for a greater contribution to the flux by older stars surrounding the central brust.

\section{Summary}

We have presented medium-resolution spectroscopy observations of 97 blue compact galaxies. These BCGs cover a range in absolute blue magnitude $-21.9<M_{B}<-14.0$; 83 BCGs have $M_{B}<-17$ mag. The spectra were obtained with the Beijing Astronomical Observatory $2.16 \mathrm{~m}$ telescope, with spectral resolution $(F W H M)$ of about $10 \AA$ and spectral range $3580 \AA-7600 \AA$. The spectrophotometry is expected to be accurate to $10 \%$.

Based on the emission and absorption lines, we measured the recession velocities of the sample galaxies. Most galaxies in the sample have velocities below $9000 \mathrm{~km} \mathrm{~s}^{-1}$; the error in recession velocity is less than $67 \mathrm{~km} \mathrm{~s}^{-1}$.

The majority of the sample galaxies are dominated by active star formation; 74 of them have typical H II regions spectra, the others are Seyfert galaxies and absorption line galaxies.

Measurement of the spectral line strengths and the results of the spectral analysis of these galaxies will be presented in the next paper of this series. We will use these data to understand the star formation history, the physical parameters governing the burst mode of star formation and chemical evolution in blue compact galaxies.

Acknowledgements. We are grateful to Dr. A. Weiss and Dr. S. Charlot for their helpful comments, constructive suggestions, and hard work of English revision for this paper. The referee Dr. D. Kunth is thanked for many useful suggestions. We also thank the BAO Chinese $2.16 \mathrm{~m}$ Telescope time allocation committee for their support of this programme and to the staff and telescope operators of the Xinglong Station of the Beijing Astronomical Observatory for their support. Especially we would like to thank Dr. J. Y. Wei and Dr. X. J. Jiang for their active cooperation that enabled all of the observations to go through smoothly. This work is supported by the Chinese National Natural Science Foundation (CNNSF 10073009). Dr. X. Kong has been financed by the Special Funds for Major State Basic Research Projects of China and the Alexander von Humboldt Foundation of Germany. 


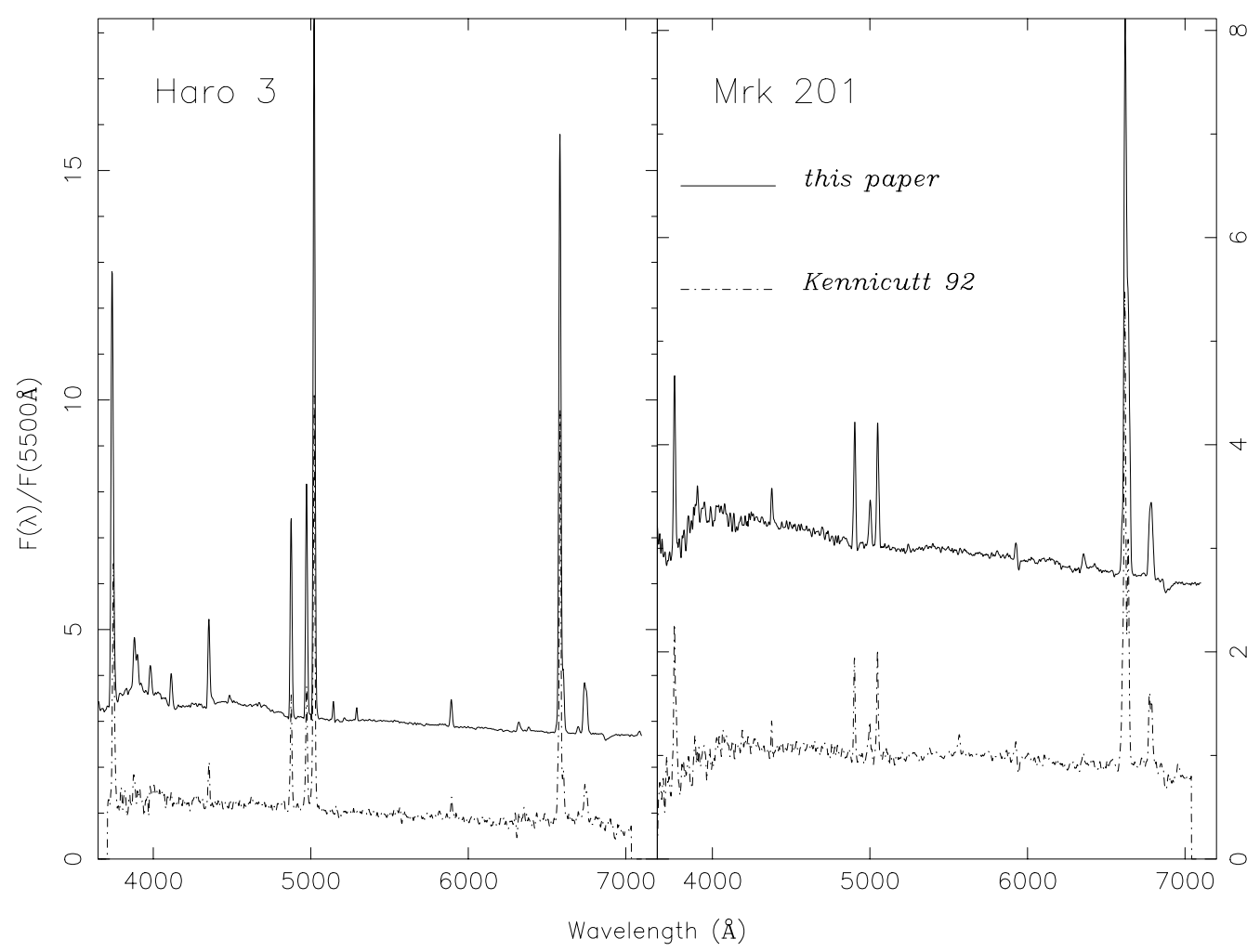

Fig. 5. b) Comparison of our spectrophotometry with Kennicutt (1992): Haro 3 and Mrk 201. The spectra have all been normalized to the flux centered on $5500 \AA$ and have been offset for clarity.

\section{References}

Cairós, L. M., Caon, N., Vílchez, J. M., et al. 2001, ApJS, 136, 393

Cardelli, J. A., Clayton, G. C., \& Mathis, J. S. 1989, ApJ, 345, 245

de Vaucouleurs, G., de Vaucouleurs, A., Corwin, Jr. H. G., et al. 1991, Third Reference Catalogue of Bright Galaxies (RC3) (Springer-Verlag: New York)

Doublier, V., Caulet, A., \& Comte, G. 1999, A\&AS, 138, 213

Filippenko, A. V. 1982, PASP, 94, 715

Gordon, D., \& Gottesman, T. S. 1981, AJ, 86, 161

Haro, G. 1956, AJ, 61, 178

Izotov, Y. I., Chaffee, F. H., \& Green, R. F. 2001, ApJ, 562, 727

Izotov, Y. I., \& Thuan, T. X. 1999, ApJ, 511, 639

Jangren, A., Bershady, M. A., Conselice, C. J., et al. 2002, ApJ, submitted

Jansen, R. A., Fabricant, D., Franx, M., et al. 2000, ApJS, 126, 331

Kinney, A. L., Bohlin, R. C., Calzetti, D., et al. 1993, ApJS, 86,5

Kennicutt, R. C. 1992, ApJS, 79, 255

Kong, X., \& Cheng, F. Z. 1999, A\&A, 351, 477

Kong, X., Zhou, X., Chen, J. S., et al. 2000, AJ, 119, 2745

Kunth, D., Lequeux, J., Mas-Hesse, J. M., et al. 1997, Rev. Mex. Astron. Astrofís., 6, 61
Kunth, D., Maurogordato, S., \& Vigroux, L. 1988, A\&A, 204, 10

Kunth, D., \& Östlin, G. 2000, A\&AR, 10, 1

Kunth, D., \& Östlin, G. 2001, ApSSS, 277, 281

Kunth, D., \& Sargent, W. L. W. 1979, A\&A, 76, 50

Markarian, B. E., Lipovetsky, V. A., Stepanian, J. A., et al. 1989, Soobshcheniya Spetsial'noj Astrofizicheskoj Observatorii, 62, 5

Mas-Hesse, J. M., \& Kunth, D. 1999, A\&A, 349, 765

Massey, P., Strobel, K., Barnes, J. V., et al. 1988, ApJ, 328, 315

Östlin, G., Amram, P., Bergvall, N., et al. 2001, A\&A, 374, 800

Pisano, D. J., Kobulnicky, H. A., Guzmán, R., et al. 2001, AJ, 122,1194

Sargent, W. L. W., \& Searle, L. 1970, ApJ, 162, L115

Terlevich, R., Melnick, J., Masegosa, J., et al. 1991, A\&AS, 91, 285

Thuan, T. X., Lipovetsky, V. A., Martin, J.-M., et al. 1999, A\&AS, 139, 1

Thuan, T. X., \& Martin, G. E. 1981, ApJ, 247, 823

Véron-Cetty, M.-P., \& Véron, P. 2001, Quasars and Active Galactic Nuclei (10th ed.), ESO Scientific Rep., 20

Zwicky, F. 1971, Catalogue of selected compact galaxies and of post-Eruptive galaxies (Guemligen: Zwicky) 